\title{
EFICIÊNCIA NUTRICIONAL DE POTÁSSIO E CRESCIMENTO DE EUCALIPTO INFLUENCIADOS PELA COMPACTAÇÃO DO SOLO(1)
}

\author{
S. R. SILVA(2), N. F. BARROS(3), R. F. NOVAIS ${ }^{(3)}$ \& P. R. G. PEREIRA(4)
}

\begin{abstract}
RESUMO
A compactação do solo por tráfico de veículos pesados altera a disponi bilidade de nutrientes para as plantas, interfere no cresci mento radicular e nos processos de fluxo de massa e de difusão, constituindo um problema no manejo florestal, no qual têm sido utilizadas máquinas de maior capacidade de carga. O objetivo deste trabalho foi avaliar a influência da compactação de solos e doses de K no crescimento e nutrição potássica de mudas de Eucalyptus camaldulensis. Utilizaram-se amostras de dois solos com texturas diferentes, em vasos sob condição de casa de vegetação, sendo os tratamentos dispostos num esquema fatorial $3 \times 4$ (densidades de solo e doses de $K$ ) para cada solo, em delineamento inteiramente casualizado, com três repetições. Amostras de dois solos, um Latossolo Vermelho argi loso ( $\mathrm{LV}_{\text {arg }}$ ) e um Latossolo Vermelho-Amarelo franco-arenoso ( $\left(\mathrm{VA}_{\mathrm{far}}\right)$, foram acondicionadas em vasos de PVC com $\mathbf{2} \mathrm{dm}^{3} \mathrm{de}$ solo e compactadas com o auxílio de uma prensa hidráulica. Para o solo argiloso, foram testadas as densidades de 0,9; 1,1 e 1,3 $\mathrm{g} \mathrm{cm}^{-3} \mathrm{e}$, para o solo franco-arenoso, de 1,3; 1,5 e 1,7 g cm-3 . As doses de potássio foram 0, 50, 100 e $150 \mathrm{mg} \mathrm{kg}^{-1}$ para os dois solos. 0 experimento foi colhido 100 dias após a emergência, tendo sido realizadas a quantificação da matéria seca, a mensuração de raízes (comprimento, diâmetro médio e superfície radicular) e as análises químicas, com vistas em determinar os teores de K no tecido vegetal e no solo. Constatou-se que, com a compactação do solo, de modo geral, o crescimento de raízes e a eficiência de utilização de $\mathrm{K}$ dimi nuíram e aumentou o diâmetro médio radicular. As doses de $\mathrm{K}$ el evaram o teor de $\mathrm{K}$ no tecido vegetal e proporcionaram aumento da matéria seca apenas nos tratamentos em que o solo foi mais compactado. Conclui-se que a aplicação de $\mathrm{K}$ em solos compactados é fundamental para o crescimento de plantas de eucalipto e que a compactação reduz o crescimento radicular e a eficiência da adubação potássica.
\end{abstract}

Termos para indexação: cresci mento de raízes, densidade do solo, transporte de nutrientes, fluxo difusivo.

\footnotetext{
(1) Parte da Tese de Mestrado do primeiro autor, apresentada à Universidade Federal de Viçosa - UFV. Trabalho apresentado na XIV Reunião Brasileira de Manejo e Conservação do Solo e da Água, em Cuiabá (MT). Recebido para publicação em novembro de 2001 e aprovado em setembro de 2002.

(2) Doutorando do Programa de Pós-Graduação em Solos e Nutrição de Plantas, Universidade Federal de Viçosa - UFV. CEP 36571 000 Viçosa (MG). Bolsista da CAPES. E-mail: sergio@solos.ufv.br

(3) Professor Titular do Departamento de Solos, UFV. Bolsista do CNPq. E-mails: nfbarros@ufv.br; rfnovais@ufv.br

(4) Professor Adjunto do Departamento de Fitotecnia, UFV. E-mail: ppereira@ufv.br
} 


\title{
SUMMARY: EUCALYPT GROWTH AND NUTRITIONAL EFFICIENCY OF POTASSIUM UNDER SOIL COMPACTION
}

\begin{abstract}
Traffic of heavy machinery during forest harvesting causes soil compaction and al ters the nutrient availability for plants, affects root growth, and the mass flow and diffusion processes, thus representing a problem for forest management. The objective of this study was to evaluatethe influence of soil compaction and potassium application on growth and $\mathrm{K}$ nutrition of Eucalyptus camaldulensis. Thestudy was carried out in pots under greenhouse conditions, using a completely randomized design with treatments in a $3 \times 4$ factorial arrangement (compaction level s and $\mathrm{K}$ rates) for each soil, with threereplicates. Samples of two soils, a clayey Red-Latosol ( $\left(V_{\text {arg }}\right)$ and a sandy Red Yellow-Latosol $\left(L V A_{\text {far }}\right)$, wereplaced in $2.0 \mathrm{dm}^{3}$ pots of PVC pipe and compacted to densities of $0.9,1.1$ and $1.3 \mathrm{~g} \mathrm{~cm}^{-3}$ for the $L V_{\text {arg }}$, and $1.3,1.5$ and $1.7 \mathrm{~g} \mathrm{~cm}^{-3}$ for the LVA $\mathrm{far}_{\text {, }}$ using a hydraulic press. Potassium rates were 0, 50, 100 and $150 \mathrm{mg} \mathrm{kg}^{-1}$, for both soi Is. Onehundred days after seedling emergence, the plant shoots were harvested and the dry weight, $\mathrm{K}$ contents in plant and soil, and root measurements (length, surface area, and mean diameter) assessed. Increasing soil compaction reduced both root and shoot growth and theK utilization efficiency, but increased the mean root diameter. Potassium application increased the plant nutrient content, but had no effect on shoot growth at low compaction levels. This study indicated that $\mathrm{K}$ application in compacted soils is essential for eucalypt growth; and that soil compaction reduces root growth and the efficiency of $\mathrm{K}$ acquisition by roots.
\end{abstract}

Index terms: root growth, bulk density, potassium transport, diffusive flux.

\section{INTRODUÇÃO}

A compactação dosolo, em ecossi stemas florestais, é, sem dúvida, um problema no manejo florestal, no qual têm sido utilizadas máquinas de maior tamanho e capacidade de carga. O tráfego de máquinas, realizado repetidas vezes na mesma área, nas operações de preparo do solo, col heita e transporte da madeira, causa estresse ao solo, em razão das forças mecânicas exercidas no sistema.

A absorção de nutrientes pelas plantas depende do crescimento e eficiência das raízes e da disponibilidade de nutrientes no solo. A disponibilidade é influenciada pela compactação do solo, já que os processos de fluxo de massa e de di fusão, responsáveis pelo transporte de nutrientes atéàs raízes, dependem da condição física do solo, particularmente de sua densidade (Costa, 1998; Novais \& Smyth, 1999). Assim, pode-se antecipar que, nessas condições, a produtividade florestal pode ser comprometida temporariamente ou de maneira permanente.

Apesar de representar um problema potencial para a nutrição mineral de florestas manejadas, poucos estudos avaliam o efeito da compactação na nutrição de eucalipto. A despeito de ser problema específico às condições de campo, estudos real izados em condições controladas podem servir para avaliar a influência da compactação no crescimento das raízes, da parte aérea ena nutrição de plantas. Nesse sentido, a compreensão dessas relações para K é de grande relevância prática, uma vez que esse nutrienteéconsiderado um dos principais limitantes do crescimento de florestas de eucalipto no Brasil (Barros et al., 1981a; Barros \& N ovais, 1996).

A falta de informações sobre a dinâmica de crescimento de raízes tem limitado o conhecimento da estrutura e do comportamento das florestas tropicais (Vance \& Nadkarni, 1992). Essa carência de conhecimento para espécies arbóreas ainda mantém uma lacuna que, se conhecida, certamente auxiliaria no entendimento do crescimento diferencial de plantas, em resposta à adubação eàs condições adversas, como a compactação do solo.

Este trabalho teve como objetivo avaliar a influência da compactação de solos e doses de K no crescimento e nutrição potássica de mudas de eucalipto.

\section{MATERIAL E MÉTODOS}

Dois Latossolos com diferentes características texturais foram utilizados: Latossolo Vermel ho $\left(\mathrm{LV}_{\mathrm{arg}}\right)$, da região de Sete Lagoas (MG) (textura muito argilosa), e Latossol o Vermelho-Amarel o $\left(\mathrm{LVA}_{\mathrm{far}}\right)$, da região de Três Marias (MG) (textura franco-arenosa). As amostras de solo foram coletadas na camada superficial $(0-20 \mathrm{~cm})$, destorroadas, homogeneizadas, secas ao ar e passadas em peneira de malha de $2 \mathrm{~mm}$ (TFSA), para posterior caracterização física e química (EMBRAPA, 1997) (Quadro 1). 
Quadro 1. Características químicas e físicas dos solos

\begin{tabular}{|c|c|c|}
\hline Característica & $\mathbf{L} \mathbf{V}_{\text {arg }}$ & LVAfar $_{\text {f }}$ \\
\hline $\mathrm{pH}^{(1)}$ & 4,6 & 4,9 \\
\hline $\mathrm{Al}^{3+}\left(\mathrm{cmol}_{\mathrm{c}} \mathrm{dm}^{-3}\right)^{(2)}$ & 1,2 & 0,4 \\
\hline $\mathrm{H}+\mathrm{Al}\left(\mathrm{cmol}_{\mathrm{c}} \mathrm{dm}^{-3}\right)^{(3)}$ & 9,6 & 2,2 \\
\hline $\mathrm{Ca}^{2+}\left(\mathrm{cmol}_{\mathrm{c}} \mathrm{dm}^{-3}\right)^{(2)}$ & 1,2 & 0,0 \\
\hline $\mathrm{Mg}^{2+}\left(\mathrm{cmol}_{\mathrm{C}} \mathrm{dm}^{-3}\right)^{(2)}$ & 0,1 & 0,0 \\
\hline$P\left(\mathrm{mg} \mathrm{dm}^{-3}\right)^{(4)}$ & 2,0 & 1,2 \\
\hline $\mathrm{K}\left(\mathrm{mg} \mathrm{dm}^{-3}\right)^{(4)}$ & 41 & 35 \\
\hline Carbono orgânico (dag kg-1) & 3,4 & 0,6 \\
\hline $\mathrm{P}_{\mathrm{REM}}\left(\mathrm{mg} \mathrm{L}^{-1}\right)^{(5)}$ & 9,3 & 46,9 \\
\hline Argila $\left(\mathrm{g} \mathrm{kg}^{-1}\right)$ & 740 & 130 \\
\hline Silte $\left(\mathrm{g} \mathrm{kg}^{-1}\right)$ & 120 & 30 \\
\hline Areia grossa $\left(\mathrm{g} \mathrm{kg}^{-1}\right)$ & 80 & 250 \\
\hline Areia fina $\left(\mathrm{g} \mathrm{kg}^{-1}\right)$ & 60 & 590 \\
\hline Densidade do solo $\left(\mathrm{g} \mathrm{cm}^{-3}\right)$ & 0,9 & 1,3 \\
\hline Capacidade de campo (\%) & 38,0 & 18,4 \\
\hline Classificação textural & $\begin{array}{l}\text { Muito } \\
\text { argilosa }\end{array}$ & $\begin{array}{l}\text { Franco } \\
\text { arenosa }\end{array}$ \\
\hline
\end{tabular}

(1) $\mathrm{pH}$ em água, relação $1: 2,5$. (2) Extrator $\mathrm{KCl} 1 \mathrm{~mol} \mathrm{~L}^{-1}$. (3) Extrator acetado de cálcio $0,5 \mathrm{~mol} \mathrm{~L}^{-1} \mathrm{pH} \mathrm{7,0.} \mathrm{(4)} \mathrm{Extrator}$ Mehlich-1. ${ }^{(5)}$ Fósforo remanescente (Alvarez V. et al., 2000).

O experimento foi realizado em casa de vegetação, sendo utilizado um fatorial $3 \times 4$ (densidades de solo e doses de K) para cada solo, com três repetições, e as unidades experimentais distribuídas inteiramente ao acaso. As densidades testadas foram 0,9; 1,1 e $1,3 \mathrm{~g} \mathrm{~cm}^{-3}$, para o $\mathrm{LV}_{\mathrm{arg}}$, e 1,3; 1,5 e $1,7 \mathrm{~g} \mathrm{~cm}^{-3}$, para $\mathrm{LVA}_{\text {far. }}$. As doses foram 0, 50, 100 e $150 \mathrm{mg} \mathrm{kg}^{-1}$ de $\mathrm{K}$ para os dois solos.

Os vasos utilizados foram tubos de PVC de $150 \mathrm{~mm}$ de diâmetro com fundo de isopor e capacidade de $2,2 \mathrm{dm}^{3}$. Calcularam-seas quantidades de TFSA de cada solo para ocupar um volume constante de $2 \mathrm{dm}^{3}$ e atingir as densidades correspondentes aos tratamentos a serem testados.

Os solos receberam, na época da adubação, quantidades de $\mathrm{Mg}$ e de Ca para fornecerem $0,4 \mathrm{cmol}_{\mathrm{c}} \mathrm{dm}^{-3}$ de $\mathrm{Mg}^{2+}$ e $1,0 \mathrm{cmol}_{\mathrm{c}} \mathrm{dm}^{-3}$ de $\mathrm{Ca}^{2+}$. Além das doses de $K$, a seguinte adubação básica foi aplicada: $100 \mathrm{mg} \mathrm{kg}^{-1}$ de $\mathrm{N}$; $200 \mathrm{mg} \mathrm{kg}^{-1}$ de $\mathrm{P}$ no $\mathrm{LVA}_{\text {far }}$ e $300 \mathrm{mg} \mathrm{kg}^{-1}$ de P no LV arg; $30 \mathrm{mg} \mathrm{kg}^{-1}$ de S; $0,82 \mathrm{mg} \mathrm{kg}^{-1}$ de $\mathrm{B}$ e $4 \mathrm{mg} \mathrm{kg}^{-1}$ de $\mathrm{Zn}$. As fontes dos elementos foram: $\left(\mathrm{MgCO}_{3}\right)_{4} \cdot \mathrm{Mg}(\mathrm{OH})_{2} \cdot 5 \mathrm{H}_{2} \mathrm{O}, \mathrm{H}_{3} \mathrm{BO}_{3}$, $\mathrm{ZnSO}_{4} .7 \mathrm{H}_{2} \mathrm{O}, \quad\left(\mathrm{NH}_{4}\right)_{2} . \mathrm{SO}_{4}, \mathrm{KNO}_{3}, \mathrm{KCl}$, $\mathrm{Ca}\left(\mathrm{H}_{2} \mathrm{PO}_{4}\right)_{2} \cdot \mathrm{H}_{2} \mathrm{O}, \mathrm{CaCl}_{2} \cdot 2 \mathrm{H}_{2} \mathrm{O}$.

A pós a adubação e homogenização, a umi dade foi elevada atéà capacidade de campo, e os sol os foram incubados por um período de dez dias. Posteriormente, realizou-se a compactação dos solos nos vasos, utilizando-se uma prensa hidráulica. Para isso, dividiu-se o solo em quatro porções iguais que foram individualmente prensadas.
Foram utilizadas aproximadamente 20 sementes de Eucalyptus camal dulensis por vaso. Oito a quinze dias após a emergência, foi realizado o desbaste, deixando-se duas plantas por vaso.

O ensaio foi desenvolvido por um período de 100 dias após a emergência das plantas, mantendose a umidade próxima à capacidade de campo, por meio de irrigações diárias com água destilada.

Após 100 dias da emergência, as plantas foram cortadas na altura de, aproximadamente, $1 \mathrm{~cm}$ acima da superfície do solo e acondicionadas em sacos de papel, e, posteriormente, colocadas em estufa com circulação forçada de ar a $65^{\circ} \mathrm{C}$, para secagem até massa constante.

Foi retirada de cada vaso, entre as duas plantas, uma amostra desolo ( $136 \mathrm{~cm}^{3}$ ) ao longo da coluna com auxílio de um trado com 3,8 cm de diâmetro interno. A amostra foi submetida a lavagem em água corrente, sobre peneira de 0,25 mm de abertura, para retirada de raízes, as quais foram utilizadas na avaliação do comprimento (L), pelo método da intercepção de linhas (Tennant, 1975), e do diâmetro médio (d), com auxílio de mi crômetro óptico acoplado a mi croscópio ocular (média de 15 leituras por amostra).

Estimou-se a densidade radicular $\left(\mathrm{cm} \mathrm{cm}^{-3}\right)$ pela divisão do comprimento radicular pelo volume de sol oamostrado (136 $\mathrm{cm}^{3}$ ). A superfície radicular (SR) foi estimada pela fórmula: $S R=\pi d L$. O valor obtido foi dividido pelo volume de solo amostrado, e o resultado expresso emam $\mathrm{am}^{-3}$ desolo. Nessa fórmula, pressupõe-se que a raiz apresente forma cilíndrica.

Os vasos foram desmontados eas raízes restantes foram retiradas mediante lavagem com água corrente, sobre peneira de 0,5 mm de abertura, e, a seguir, foram secas de modo idêntico à parte aérea.

As amostras de tecido vegetal foram moídas em moinho tipo Wiley de aço inoxidável, passadas em peneira de 0,1 mm, para subseqüente digestão nítrico-perclórica (J ohnson \& Ulrich, 1959) para determinação de $K$, por fotometria de chama.

A partir do teor de $K$ na planta (parte aérea + sistema radicular) em todos os tratamentos, foram calculados os índices de eficiência: (a) Eficiência de utilização deK (Siddiqi \& Glass, 1981): (matéria seca total produzida) $2 /($ conteúdo de K na planta); (b) Eficiência de absorção ou razão de eficiência radicular de K (Batten, 1992): (conteúdo de K na planta)/(superfície radicular). Os valores de superfície radicular utilizados no cálculo de eficiência de absorção foram obtidos pela correção dos valores de superfície radicular no solo amostrado (SR) para o volume total de sol o no vaso.

Os dados foram submetidos à análise de variância em del ineamento intei ramente casual izado, usandose o programa SAE G (Sistema deAnálise Estatística e Genética). Ajustaram-se os model os de regressão linear, quadrático e quadrático base raiz quadrada. Para a escolha dos modelos, os coeficientes foram 
testados pelo teste de $\mathrm{F}$ a 1 e $5 \%$, com base no quadrado médio do resíduo da análise de variância. Finalmente, para dois ou mais modelos com coeficientes significativos, a sel eção foi feita com base no maior $\mathrm{R}^{2}$ ajustado. Foram determinadas as doses de K responsáveis por 90 \% da produção máxima estimada de matéria seca total.

\section{RESULTADOS E DISCUSSÃO}

\section{Produção de matéria seca}

As doses deK não influíram significativamentena produção de matéria seca de raízes nas densidades de 0,9 e $1,3 \mathrm{~g} \mathrm{~cm}^{-3}$ no $\mathrm{LV}_{\text {arg }}$ (Figura 1 ). Contudo, o comportamento de doses de $\mathrm{K}$ foi quadrático em todas as densidades testadas no $L A_{f a r}$ e na densidadeintermediária do primeiro solo (Figura 1).
Foi baixa a resposta em produção de matéria seca da parte aérea e total às doses de $K$ nas densidades de 0,9 e $1,1 \mathrm{~g} \mathrm{~cm}^{-3}$ noLV $_{\text {arg }}$ e $1,3 \mathrm{~g} \mathrm{~cm}^{-3}$ noLVA $_{\text {far }}$. No entanto, em densidades superiores $\left(1,3 \mathrm{~g} \mathrm{~cm}^{-3}\right.$ no $\mathrm{LV}_{\text {arg }}$ e 1,5 e $1,7 \mathrm{~g} \mathrm{~cm}^{-3}$ no $\mathrm{LVA}_{\text {far }}$ ), houve maior intensidade de resposta às doses de K (Figura 1).

É interessante destacar a resposta positiva ao $\mathrm{K}$ nos tratamentos correspondentes aos maiores níveis de compactação do solo. Nessa situação, a adubação potássica mostrou-se necessária para reverter ou amenizar os efeitos danosos da compactação no fluxo difusivo de K e no crescimento radicular.

$\mathrm{O}$ aumento do fluxo difusivo de $\mathrm{K}$ causado pela fertilização potássica contribuiu para elevar a absorção do nutriente (Figura 2), restabel ecendo parcialmente o crescimento das plantas afetado pela compactação do solo. No entanto, destaca-se que, mesmo para a maior dose de $K$, a produção de matéria seca nos tratamentos com maiores valores
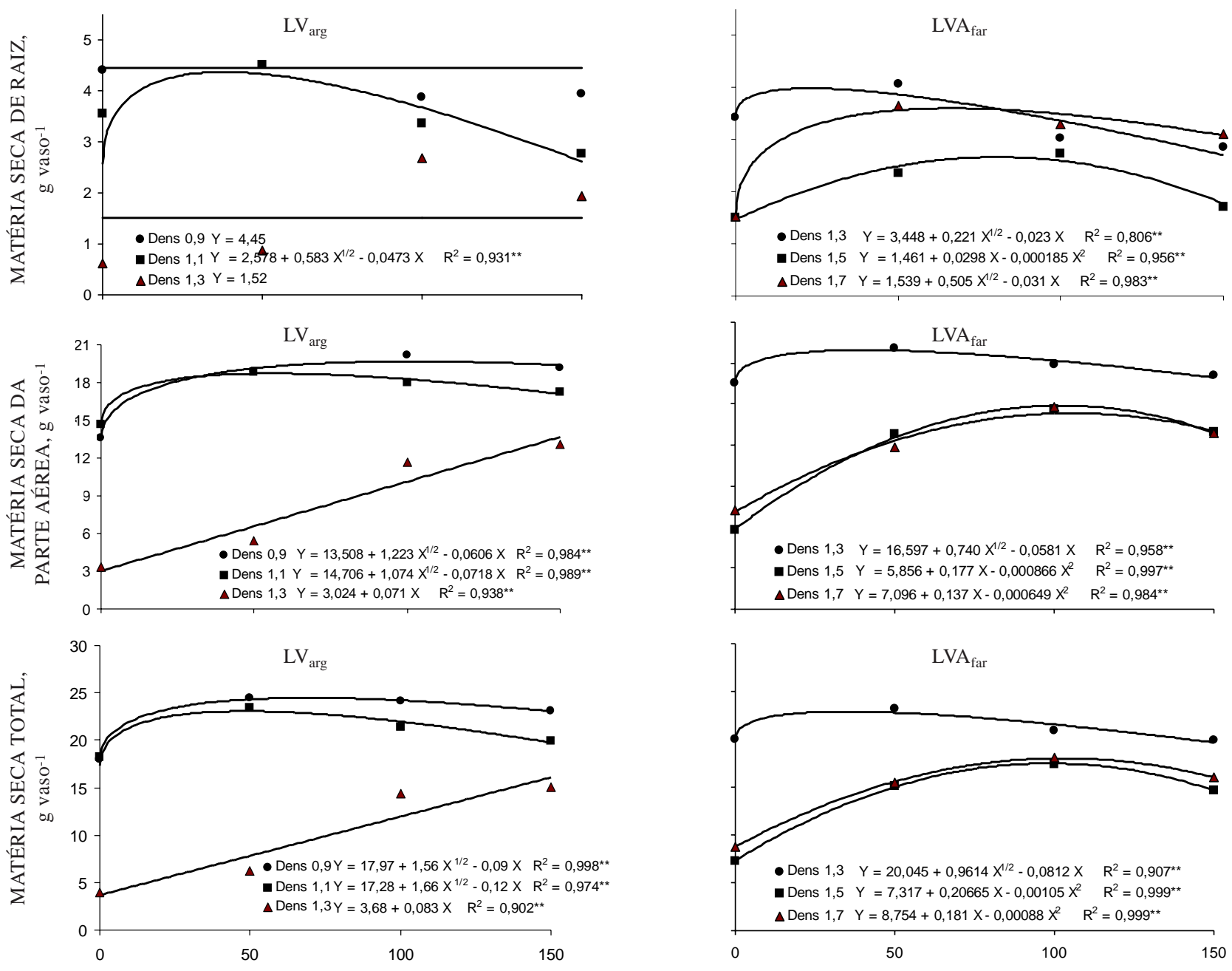

DOSE DE K, mg kg-1

Figura 1. Matéria seca de raiz, parte aérea e total de plantas de E. camaldulensis, cultivadas em dois Latossolos de texturas muito argilosa ( $\mathrm{LV}_{\mathrm{arg}}$ ) e franco-arenosa ( $L \mathrm{VA}_{\mathrm{far}}$ ), com diferentes densidades e doses de potássio. 
de densidade do solo não foi completamente restabelecida. Isto podeser atribuído, dentre outras possibilidades, ao fato de a compactação do solo restringir fortemente a difusão de outros elementos como o P e o Zn (N ovais \& Smyth, 1999).

A ausência ou pequena resposta às doses de $K$, observada nos tratamentos com menores densidades do solo, pode ser atribuída ao teor inicial de $\mathrm{K}$ por Mehlich-1 de 41 e $35 \mathrm{mg} \mathrm{dm}^{-3}$, nos solos $L_{\text {arg }}$ eLVA $_{\text {far }}$, respectivamente. Esses teores são bem superiores ao nível crítico de $\mathrm{K}$ no solo de $10 \mathrm{mg} \mathrm{dm}^{-3}$, estabelecido por Novais et al. (1980), para o crescimento inicial de mudas de duas espécies de eucal ipto em viveiro e, ainda, ao val or de $7 \mathrm{mg} \mathrm{dm}^{-3}$, encontrado por Barros et al. (1981b) para mudas de E. grandis em 10 solos.

Utilizando as equações de regressão (Figura 1), foram obtidas as doses de K correspondentes a $90 \%$ da máxima produção estimada de matéria seca
(Quadro 2). Quantoà produção dematéria seca total, essas doses variaram no $L_{\text {arg }}$ entre 6,6 e $130,5 \mathrm{mg} \mathrm{kg}^{-1}$ de $\mathrm{K}$ e entre 0,4 e 57,6 $\mathrm{mg} \mathrm{kg}^{-1}$ de $\mathrm{K}$ no LVA far. E interessante observar que as maiores doses de $K$ são especialmente importantes nos mai ores níveis de compactação, indicando o aumento do nível crítico de $\mathrm{K}$ no solo.

A produção estimada de $90 \%$ da matéria seca total, para as distintas densidades do solo, variou em mais de 51 e $27 \%$, respectivamente, no $L_{\text {arg }} \mathrm{e}$ $\mathrm{LVA}_{\mathrm{far}}$, tendo a compactação restringido a produção de matéria seca de raízes em 2,8 vezes na densidade $1,3 \mathrm{~g} \mathrm{~cm}^{-3}$, noLV $\mathrm{arg}$, e em 1,5 na densidade $1,5 \mathrm{~g} \mathrm{~cm}^{-3}$, no LVA far (Quadro 2).

\section{Teor de potássio na planta}

Houve aumento do conteúdo de $\mathrm{K}$ na raiz e na parte aér ea das plantas, considerando as doses de K aplicadas nos dois sol os estudados (Figura 2).
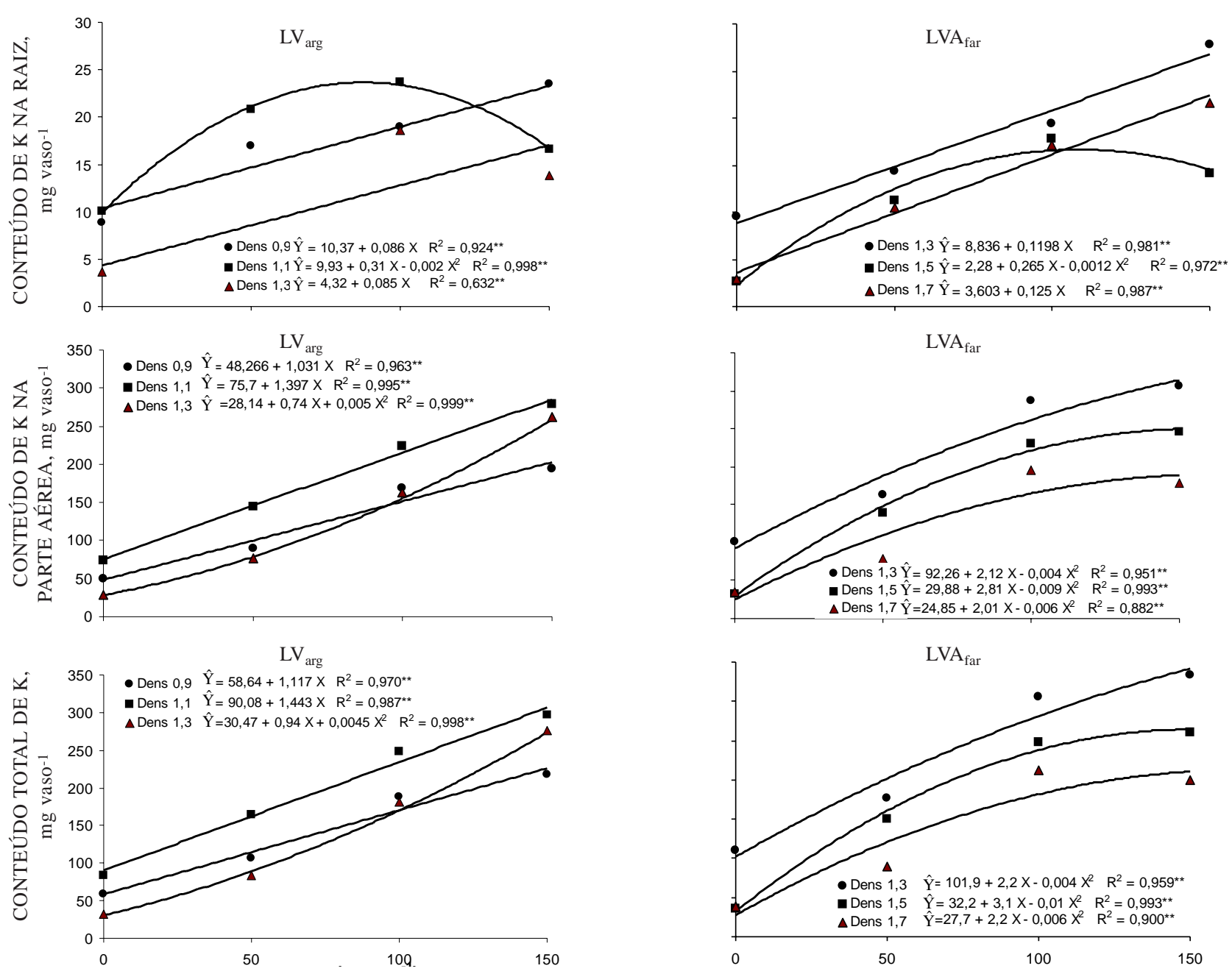

DOSE DE K, $\mathrm{mg} \mathrm{kg}^{-1}$

Figura 2. Conteúdo de $K$ na raiz, parte aérea e total de plantas de $E$. camaldulensis, cultivadas em dois Latossolos de texturas muito argilosa ( $L V_{\text {arg }}$ ) e franco-arenosa $\left(L V A_{f a r}\right)$, com diferentes densidades e doses de potássio. 
Quadro 2. Produção de matéria seca de raízes, da parte aérea e total correspondente a $90 \%$ da máxima esti mada, produção relativa total e doses de potássio necessárias para obter $\mathbf{9 0} \%$ da produção máxima de matéria seca total de plantas de E. camaldulensis, cultivadas em dois Latossolos de texturas muito argi losa $\left(\mathrm{LV}_{\mathrm{arg}}\right)$ e franco-arenosa $\left(\mathrm{LVA}_{\mathrm{far}}\right)$, com diferentes densidades e doses de potássio

\begin{tabular}{|c|c|c|c|c|c|}
\hline \multirow{2}{*}{ Densidade do solo } & \multicolumn{4}{|c|}{ Produção de matéria seca } & \multirow{2}{*}{ Dose de $K^{(1)}$} \\
\hline & Raízes & Parte aérea & Total & Produção relativa & \\
\hline \multirow[t]{2}{*}{$\mathrm{g} \mathrm{cm}^{-3}$} & \multicolumn{3}{|c|}{ g por vaso } & $\%$ & $\mathrm{mg} \mathrm{kg}^{-1}$ \\
\hline & \multicolumn{3}{|c|}{ LV } & & \\
\hline 0,9 & 4,0 & 17,7 & 21,7 & 100 & 10,4 \\
\hline 1,1 & 3,9 & 16,8 & 20,7 & 94 & 6,6 \\
\hline \multirow[t]{2}{*}{1,3} & 1,4 & $12,3^{(2)}$ & $13,7^{(2)}$ & 65 & 130,5 \\
\hline & \multicolumn{4}{|c|}{$L V A_{\text {far }}$} & \\
\hline 1,3 & 3,6 & 17,1 & 20,7 & 100 & 0,4 \\
\hline 1,5 & 2,4 & 13,4 & 15,8 & 76 & 57,6 \\
\hline 1,7 & 3,2 & 12,9 & 16,2 & 78 & 57,2 \\
\hline
\end{tabular}

(1) Doses de K para obtenção de $90 \%$ da produção máxima de matéria seca total. ${ }^{(2)}$ Correspondente a $90 \%$ da produção obtida com a maior dose de $\mathrm{K}$ utilizada.

O maior nível de compactação nos dois solos acarretou decréscimo do conteúdo de K na raiz e na parte aérea das plantas. I sso pode ser atribuído à redução da difusão $K$ e ao efeito prejudicial sobre o crescimento radicular, resultando em menor absorção do nutriente.

Costa (1998), estudando a difusão de K como variável da compactação e da umidade do solo, verificou que seus val ores diminuíram em todos os níveis de umidade e nos três sol os estudados, quando se promoveu a compactação.

No LV ${ }_{\text {arg, }}$ as plantas cultivadas na densidade intermediária $\left(1,1 \mathrm{~g} \mathrm{~cm}^{-3}\right)$ apresentaram conteúdo de $\mathrm{K}$ superior àquele presente nas plantas cultivadas na menor densidade $\left(0,9 \mathrm{~g} \mathrm{~cm}^{-3}\right)$. Considerando que nas diferentes doses testadas praticamente não houve diferença quanto à produção de matéria seca total nessas densidades (Figura 1 ), infere-se que 0 maior teor de $\mathrm{K}$ na densidade $1,1 \mathrm{~g} \mathrm{~cm}^{-3}$ é resultado da maior absorção do nutriente.

Com o aumento da densidade do solo, a difusão aumenta até atingir um máximo e depois decresce. Esse aumento deve-se à maior aproximação entre as partículas do solo e as raízes, o que favorece a continuidade do filme de água e diminui a distância que oíon deve percorrer atéal cançar a raiz (Oliveira et al., 1998).

\section{Eficiência de utilização de $K$}

A eficiência de utilização de potássio foi maior nas duas primeiras doses de potássio ( 0 e $50 \mathrm{mg} \mathrm{kg}^{-1}$ ) (exceção na densidade $1,3 \mathrm{~g} \mathrm{~cm}^{-3}$, no $L_{\text {arg }}$ ), apresentando, em seguida, dedínio com o aumento das doses do nutriente (Quadro 3). I sso concorda com resultados de Galo (1993), que verificou, em experimento com eucal ipto no campo, que a eficiência de utilização de K na produção de tronco e de copa diminuiu com a elevação da dose do nutriente aplicada. Silva et al. (1996), estudando espécies florestais nativas, também constataram que a eficiência de utilização de K reduziu com o aumento da disponibilidade desse nutriente. Em geral, o aumento da concentração do nutriente na solução do solo leva à sua maior absorção pelas plantas. Quando a taxa de crescimento destas é menor do que a taxa de absorção, percebe-se redução na eficiência de uso do nutriente em questão.

Quadro 3. Eficiência de utilização de potássio por plantas de E. camaldulensis, cultivadas em dois Latossolos de texturas muito argilosa $\left(L_{\mathrm{arg}}\right)$ e franco-arenosa ( $L$ VA $_{\text {far }}$ ), com diferentes densidades e doses de potássio

\begin{tabular}{|c|c|c|c|c|}
\hline \multirow{2}{*}{ Densidade do solo } & \multicolumn{4}{|c|}{ Dose de K (mg kg-1) } \\
\hline & $\mathbf{0}$ & 50 & 100 & 150 \\
\hline \multirow[t]{2}{*}{$\mathrm{g} \mathrm{cm}^{-3}$} & \multicolumn{4}{|c|}{$-g^{2} \mathrm{mg}^{-1}$ de $\mathrm{K}$} \\
\hline & \multicolumn{4}{|c|}{$\mathrm{LV}_{\text {arg }}$} \\
\hline 0,9 & 5,5 & 5,6 & 3,1 & 2,4 \\
\hline 1,1 & 4,0 & 3,3 & 1,8 & 1,3 \\
\hline \multirow[t]{2}{*}{1,3} & 0,5 & 0,5 & 1,1 & 0,8 \\
\hline & \multicolumn{4}{|c|}{$L V A_{f a r}$} \\
\hline 1,3 & 3,6 & 3,0 & 1,4 & 1,2 \\
\hline 1,5 & 1,5 & 1,5 & 1,2 & 0,8 \\
\hline 1,7 & 2,0 & 2,7 & 1,5 & 1,3 \\
\hline
\end{tabular}


Como o teor inicial de $\mathrm{K}$ nos solos era relativamentealto(Quadro 1), os valores observados para eficiência de utilização na ausência ena menor dose do fertilizante possivel mente encontravam-se próximos ao máximo. Essa consideração está de acordo com resultados deSiddiqi \& Glass (1981), que relataram tendência quadrática na eficiência de utilização de $\mathrm{K}$, o que foi atribuído à semelhança entre as curvas de crescimento e de atuação enzimática.

A eficiência de utilização observada na densidade $1,3 \mathrm{~g} \mathrm{~cm}^{-3}$ no $\mathrm{LV}_{\text {arg }}$ (Quadro 3) foi decorrente da compactação, que proporcionou acentuada redução na produção de matéria seca nas menores doses de $\mathrm{K}\left(0\right.$ e $50 \mathrm{mg} \mathrm{kg}^{-1}$ ) (Figura 1 ).

A restrição à produção de matéria seca, proporcionada pela compactação do solo, levou à redução da eficiência de utilização de potássio, sendo esse efeito observado, com mais clareza, no $L_{\text {arg. }}$

\section{Características radiculares e eficiência de absorção}

Em resposta ao aumento da compactação, houve decréscimo da densidade de raízes nos dois solos, com exceção na densidade $1,7 \mathrm{~g} \mathrm{~cm}^{-3}$, no $\operatorname{LVA}_{\mathrm{far}}$ (Figura 3). O decréscimo do comprimento de raízes em maiores níveis de compactação do solo foi relatado por Nadian et al. (1996), em plantas de trevo, e também por Misra \& Gibbons (1996), em plantas jovens de eucalipto. A diminuição na extensão de raízes em solos compactados está relacionada com vários fatores. Em solos secos, o aumento da resistência mecânica e o decréscimo do potencial de água no solo podem ser os mais importantes. Em contrapartida, em sol os úmidos, a deficiência de oxigênio e a acumulação de etileno e fitotoxinas são os fatores principais (Marschner, 1995). Segundo Russel \& Russel (1964), citados por Borges et al. (1997), é possível que a diminuição na expansão do sistema radicular seja resultante do acúmulo de $\mathrm{CO}_{2}$ e da baixa difusão de oxigênio. Quando a concentração de $\mathrm{O}_{2}$ é muito baixa, pode ocorrer redução na pressão de turgescência da célula ou, mesmo, maior resistência da parede celular ao alongamento.

Verificou-se que, nas maiores densidades, houve o aumento do diâmetro médio de raízes nos dois sol os e o decréscimo da superfície radicular no $L_{\text {arg }}$ (Figura 3). Normalmente, quando crescem em sol os com alta resistência, as raízes apresentam diâmetro aumentado (Rosolem et al ., 1994). Shierlaw \& Alston (1984) e Nadian et al. (1996) observaram aumento do diâmetro de raízes principais e laterais de primeira ordem, com o aumento da compactação do solo. Aumento no diâmetro radicular tem sido atribuído à ampliação do córtex, no qual as células tornam-se mais curtas na direçãolongitudinal, mas ficam largas na transversal (Atwell, 1988). Na camada compactada, as raízes sofrem uma série de modificações, tanto de ordem fisiológica quanto morfológica, tornando-se mais curtas, grossas, escamosas e com parede celular grossa, revelando maior taxa de respiração e maior resistência estrutural, ou seja, menor maleabilidade para se desviarem daqueles pontos de maior resistência no solo. Tais características são próprias de raízes que crescem em ambiente redutor, podendo a compactação causar drenagem deficiente no sol o e, conseqüentemente, situação de anaerobi ose (Baligar et al., 1975; Materechera et al., 1992; Alvarenga et al., 1996; Misra \& Gibbons, 1996).

O aumento do diâmetro radicular podefavorecer a absorção de $\mathrm{K}$ por unidade de comprimento radicular em sol os compactados. Peterson \& Barber (1981) observaram que o aumento do diâmetro radicular de 0,34 para $0,44 \mathrm{~mm}$ aumentou o influxo máximo de $\mathrm{K}$ de 4,6 para 7,1 pmol $\mathrm{cm}^{-2} \mathrm{~s}^{-1}$.

O diâmetro de raízes é uma característica importante quanto à sua capacidade de penetração em solos compactados, uma vez que isto ocorrerá quando o diâmetro dos poros do sol o for maior que o diâmetro radicular, ou menor, se esses não se apresentarem rígidos. Assim, a compactação do solo, ao aumentar o diâmetro radicular, pode reduzir o volume de solo explorado pela raízes (Dolan et al., 1992).

As doses de $\mathrm{K}$ proporcionaram aumento da densidade de raízes e da superfície radicular no LVA $_{\text {far }}$, exceto para esta característica na densidade

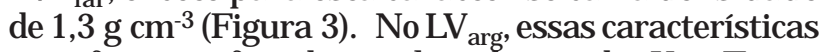
não foram afetadas pelas doses de K. Esses resultados podem ser explicados pel os teores de $\mathrm{K}$ dos solos e pelo efeito da compactação no fluxo difusivo do nutriente. NoLV ${ }_{\text {arg }}$ o oteor deK disponível inicial de $41 \mathrm{mg} \mathrm{dm}^{-3}$ (Quadro 1) e ofinal (Quadro 4) foram suficientes para manter o crescimento radicular das plantas. J á no LVA far, o teor inicial de $\mathrm{K}$ do solo foi insuficiente para sustentar o crescimento radicular e, possivel mente, para manter o fluxo difusivo de K. Neste solo, mesmo com a aplicação das doses mais elevadas, o teor de $\mathrm{K}$ ao final do experimento ficou abaixo dos níveis críticos estabel ecidos por Novais et al. (1980) e por Barros et al. (1981b) na maioria das situações.

Pouca informação está disponível sobre os efeitos dos teores de $\mathrm{K}$ do sol o no crescimento e morfologia das raízes. Assim, a variação do crescimento e das característi cas radiculares observadas entre os sol os carece de estudos mais detal hados para se al cançar um melhor entendimento dos processos envolvidos nessa dinâmica.

Houve aumento da razão de eficiência radicular (RER) de K com a aplicação das doses deK em todas as densidades nos dois solos (Figura 3 ). Tal comportamento refl ete o aumento da disponi bilidade do nutriente na solução do solo dependente da fertilização potássica. 

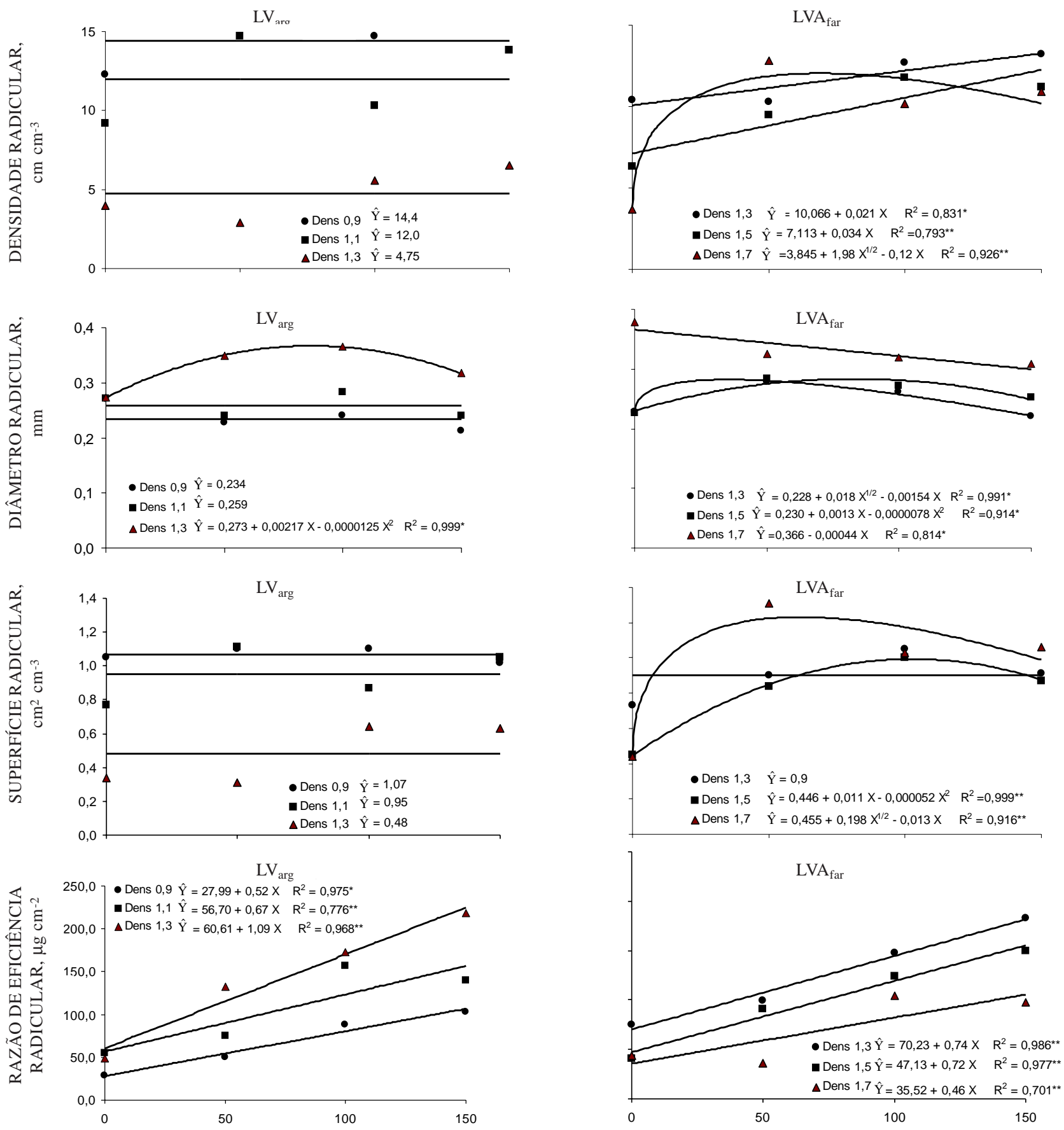

DOSE DE K, $\mathrm{mg} \mathrm{kg}^{-1}$

Figura 3. Densi dade, diâmetro e superfície radiculares e razão de eficiência radicular de K de plantas de E. camaldul ensis, cultivadas em dois Latossolos de texturas muito argilosa ( $\left.L_{\text {arg }}\right)$ e franco-arenosa $\left(\right.$ LVA $\left._{\text {far }}\right)$, com diferentes densidades e doses de potássio.

A RER de $K$ no $L V_{\text {arg }}$ apresentou valores crescentes com o aumento da densidade do solo, em conseqüência da redução do crescimento radicular e da tendência de aumento do teor de K na planta, proporcionados pela compactação do solo. E ntretanto, foi verificado efeito contrário no $L V A_{f a r}$, havendo decréscimo da RER de $\mathrm{K}$ com o aumento da densidade do solo. O baixo teor final de $\mathrm{K}$ no LVA $_{\text {far }}$ (Quadro 4), os maiores valores de conteúdo deK nas plantas cultivadas em menores densidades nesse solo (Figura 2) e os maiores valores de superfície radicular nos tratamentos com maiores níveis de compactação (Figura 3) foram a causa da mudança de comportamento observada nesse solo. 
Quadro 4. Teores de potássio em dois L atossolos de texturas muito argilosa $\left(L V_{\text {arg }}\right)$ e francoarenosa $\left(L V_{f a r}\right)$, influenciados por doses de potássio, ao término do experimento

\begin{tabular}{lccccc}
\hline \multirow{2}{*}{ Solo } & $\begin{array}{c}\text { Densidade } \\
\text { do solo }\end{array}$ & $\mathbf{0}$ & $\mathbf{5 0}$ & $\mathbf{1 0 0}$ & $\mathbf{1 5 0}$ \\
\cline { 3 - 6 } & $\mathrm{g} \mathrm{cm}^{-3}$ & \multicolumn{4}{c}{$\mathrm{mg} \mathrm{kg}^{-1}$} \\
\cline { 3 - 6 } LVarg & 0,9 & 9,9 & 11,4 & 13,2 & 15,0 \\
& 1,1 & 7,9 & 15,5 & 17,0 & 27,6 \\
& 1,3 & 12,3 & 19,5 & 30,8 & 44,9 \\
& & & & & \\
LVA $_{\text {far }}$ & 1,3 & 3,0 & 3,5 & 4,7 & 14,6 \\
& 1,5 & 1,7 & 3,0 & 3,9 & 10,8 \\
& 1,7 & 2,3 & 3,4 & 3,8 & 7,8 \\
\hline
\end{tabular}

Coeficiente de variação no $L V_{\text {arg }}=26,7 \%$ e no $L V A_{\text {far }}=37,9 \%$.

\section{CONCLUSÕES}

1. A compactação do solo restringiu o crescimento eaumentou o diâmetro médio de raízes de eucalipto, bem como reduziu o acúmulo de $\mathrm{K}$ na planta, diminuindo a eficiência de utilização de $\mathrm{K}$ e a eficiência da adubação potássica.

2. As doses de potássio el evaram o teor de $\mathrm{K}$ no tecido vegetal e proporcionaram aumento de produção de matéria seca apenas nos maiores níveis de compactação, el evando a razão de eficiência radicular de $\mathrm{K}$.

\section{LITE RATURA CITADA}

ALVARENGA, R.C.; COSTA, L.M.; MOURA FILHO, W. \& REGAZZI, A.J . Crescimento de raízes de leguminosas em camadas de solo compactadas artificialmente. R. Bras. Ci. Solo, 20:319-326, 1996.

ALVAREZ V., V.H.; NOVAIS, R.F.; DIAS, L.E. \& OLIVEIRA, J.A. Determinação e uso do fósforo remanescente. B. Inf. SBCS, 25:27-32, 2000.

ATWELL, B.J. Physiological responses of lupin roots to soil compaction. Plant Soil, 111:277-281, 1988.

BALIGAR, V.C.; NASH, V.E.; HARE, M.L. \& PRICE J r., J .A. Soybean root anatomy as influenced by soil bulk density. Agron. J ., 67:842-844, 1975.

BARROS, N.F.; BRAGA, J.M.; BRANDI, R.M. \& DEFELIPO, B.V. Produção de eucalipto em solos de cerrado em resposta à aplicação de NPK e de B e Zn. R. Árv., 5:90-103, 1981a.

BARROS, N.F.; GOMES, J.M.; NOVAIS, R.F. \& NEVES, J.C.L. Níveis críticos de Ca e $K$ no solo para o crescimento de mudas de Eucalyptus grandis. In: CONGRESSO BRASI LEIRO DE CIÊNCIA DO SOLO, 18.; Salvador, 1981. Anais. Salvador, Sociedade Brasileira de Ciência do Solo, 1981b. p.92.
BARROS, N.F. \& NOVAIS, R.F. Eucalypt nutrition and fertilizer regimes in Brazil. In: ATTIWILL, P.M. \& ADAMS, M.A., eds. Nutrition of eucalypts. Collingwood, CSI RO Publishing, 1996. p.335-355.

BATTEN, G.D. A review of phosphorus efficiency in wheat. Plant Soil, 146:163-168, 1992.

BORGES, E.N.; LOMBARDI NETO, F.; CORRÊA, G.F. \& COSTA L.M. Misturas de gesso e matéria orgânica alterando atributos físicos de um Latossolo com compactação simulada. R. Bras. Ci. Solo, 21:125-130, 1997.

COSTA, J .P.V. Fluxo difusivo de fósforo e de potássio em Latossolos. Viçosa, Universidade Federal de Viçosa, 1998. 67p. (Tese de Doutorado)

DOLAN, M.S.; DOWDY, R.H.;VOORHEES, W.B.; OHNSON,J .F. \& BIDWELL-SCHRADER, A.M. Corn phosphorus and potassium uptake in response to soil compaction. Agron. J ., 84:639-642, 1992.

EMPRESA BRASILEIRA DE PESQUISA AGROPECUÁRIA EMBRAPA. Centro Nacional de Pesquisa de Solos. Manual de métodos de análise de solo. 2.ed. Rio de J aneiro, 1997. $212 p$.

GALO, M.V. Resposta do eucalipto à aplicação de potássio em solo de cerrado. Viçosa, Universidade Federal de Viçosa, 1993. 40p. (Tese de Mestrado)

J OHNSON, C.M. \& ULRICH, A. Analytical methods for use in plants analyses. Los Angeles, University of California, 1959. p.32-33. (Bulletin, 766)

MARSCHNER, H. Mineral nutrition of higher plants. 2.ed. San Diego, Academic Press, 1995. 889p.

MATERECHERA, S.A.;ALSTON,A.M.;KIRBY,J .M. \& DEXTER, A.R. Influence of root diameter on the penetration of seminal roots into a compacted soil. Plant Soil, 144:297303, 1992

MISRA, R.K. \& GIBBONS, A.K. Growth and morphology of eucalypt seedling-roots, in relation to soil strength arising from compaction. Plant Soil, 182:1-11, 1996.

NADI AN, H.; SMITH, S.E.; ALSTON, A.M \& \& MURRAY, R.S.The effect of soil compaction on growth and $P$ uptake by Trifolium subterraneum: interactions with mycorrhizal colonisation. Plant Soil, 182:39-49, 1996.

NOVAIS, R.F.; REGO, A.K. \& GOMES, J.M. Nível crítico de potássio no solo e na planta para o crescimento de mudas de E. grandis W. Hill ex-Maiden e de E. cloeziana F. Muell. R. Árv., 4:14-23, 1980.

NOVAIS, R.F. \& SMYTH, T.J . Fósforo em solo e planta em condições tropicais. Viçosa, Universidade Federal deViçosa, 1999. 399p.

OLIVEIRA, C.V.; BAHIA, V.G. \& PAULA, M.B. Compactação do solo devido à mecanização florestal: causas, efeitos e práticas de controle. Inf. Agropec., 191:46-48, 1998.

PETERSON, W.R. \& BARBER, S.A. Soybean root morphology and K uptake. Agron. J ., 73:316-319, 1981.

ROSOLEM, C.A.; ALMEIDA, A.C.S. \& SACRAMENTO, L.V.S.S. Sistema radicular e nutrição da soja em função da compactação do solo. Bragantia, 53:259-266, 1994.

SHIERLAW, J \& \& ALSTON, A.M. Effects of soil compaction on root growth and uptake of phosphorus. Plant Soil, 77:15-28, 1984. 
SIDDIQI, M.Y. \& GLASS, A.D.M. Utilization index: a modified approach to the estimation and comparison of nutrient utilization efficiency in plants. J. Plant Nutr., 4:289-302, 1981.

SILVA, I.R.; FURTINI NETO, A.E.; VALE, F.R. \& CURI, N. Eficiência nutricional para potássio em espécies florestais nativas. R. Bras. Ci. Solo, 20:257-264, 1996.
TENNANT, D.A. A test of a modified line intersect method of estimating root lenght. J. Ecol., 63:995-1001, 1975.

VANCE, E.D. \& NADKARNI, N.M. Root biomass distribuition in a moist tropical Montane forest. Plant Soil, 142:31-39, 1992. 